\title{
Strain rate effects for spallation of concrete
}

\author{
Ulrich Häussler-Combe ${ }^{a}$, Evmorfia Panteki ${ }^{b}$, and Tino Kühn ${ }^{c}$ \\ Institut für Massivbau, Technische Universität Dresden, 01062 Dresden, Germany
}

\begin{abstract}
Appropriate triaxial constitutive laws are the key for a realistic simulation of high speed dynamics of concrete. The strain rate effect is still an open issue within this context. In particular the question whether it is a material property - which can be covered by rate dependent stress strain relations - or mainly an effect of inertia is still under discussion. Experimental and theoretical investigations of spallation of concrete specimen in a Hopkinson Bar setup may bring some evidence into this question. For this purpose the paper describes the VERD model, a newly developed constitutive law for concrete based on a damage approach with included strain rate effects [1]. In contrast to other approaches the dynamic strength increase is not directly coupled to strain rate values but related to physical mechanisms like the retarded movement of water in capillary systems and delayed microcracking. The constitutive law is fully triaxial and implemented into explicit finite element codes for the investigation of a wide range of concrete structures exposed to impact and explosions.

The current setup models spallation experiments with concrete specimen [2]. The results of such experiments are mainly related to the dynamic tensile strength and the crack energy of concrete which may be derived from, e.g., the velocity of spalled concrete fragments. The experimental results are compared to the VERD model and two further constitutive laws implemented in LS-Dyna. The results indicate that both viscosity and retarded damage are required for a realistic description of the material behaviour of concrete exposed to high strain effects [3].
\end{abstract}

\section{Introduction}

Experimental observations demonstrate an increase in concrete strength with rising strain rates. This phenomenon is referred to as strain rate effect and is particularly noticeable in tension. The strength increase is normally characterized by the Dynamic Increase Factor (DIF), which expresses the ratio of dynamic strength to the corresponding quasistatic value. In practise this aspect is often relevant for concrete structures exposed to traffic accidents, earthquakes, rockfalls and similar cases of impact and explosions.

A summary of experimental work on the material behavior of concrete under high strain rates is given in Fig. 1 for compression [4] and tension [6]. Layed on logarithmic axes determined DIF values can be approximated by a bilinear curve. DIF-values of up to 2 for strain rates up to $\approx 10 \mathrm{~s}^{-1}$ can be approximated by the first flat branch. Strain rates above $10 \mathrm{~s}^{-1}$ can be approximated by the second steeper branch with DIF-values up to 10 for stain rates of $\approx 100 \mathrm{~s}^{-1}$.

Corresponding on the assumption the two branches of the DIF-diagram are in connection with different physical mechanisms, the VERD model was developed. Under the terms of this concept, the flat branch results from pore water bound in the capillary systems of cement. Viscous effects take place during the deformation of pores and allow the material to withstand higher stresses under high strain rate conditions [7]. In this regard the second steeper

\footnotetext{
a e-mail: ulrich.haeussler-combe@tu-dresden.de

b e-mail: evmorfia.panteki@tu-dresden.de

c e-mail: tino.kuehn@tu-dresden.de
}

branch is contributed to retarded damage $[8,9]$. Cracks cannot spread arbitrarily fast, which implies that crack speeds below the materials wave speed permit the lowering of stresses even before the formation of cracks.

The paper examines the VERD model and evaluates their suitability for the modelling of concrete behavior under high strain rates in contrast to two further constitutive models implemented in LS-Dyna. The VERD model was added to the program with a user-defined subroutine. The KCC (Karagozian \& Case Concrete model) and the RHT (Riedel, Hiermaier and Thoma) model were chosen for comparative reasons, as they are among the most commonly used concrete constitutive models in LS-Dyna. Both are phenomenological and provide a wide range of parameters which ideally can be adjusted to reproduce the desired concrete behavior. In terms of simplification the option of automatic parameter generation is given. This approach has been practiced here and its suitability as a first estimate was tested. The basic required parameter was the unconfined compressive strength, which was set to $30 \mathrm{~N} / \mathrm{mm}^{2}$.

\section{Homogenous behavior}

Main comparative analysis of the material behavior is done on the basis of the Split Hopkinson Bar. Prior to that homogeneous material behavior is modelled and evaluated with a one element setup using a 8-node cube. Structural inertia effects are minimized by a relatively short edge length of $1 \mathrm{~mm}$. The load is applied with a prescribed displacement of the four upper nodes, as shown in Fig. 2. This leads to a uniaxial stress state and controllable strain rates. Quasi static strengths arise for a strain rate of 


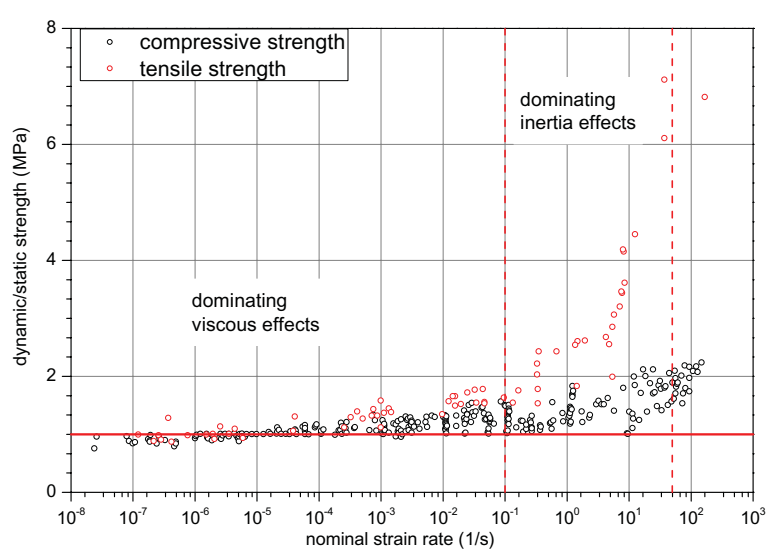

Figure 1. DIF-diagram.

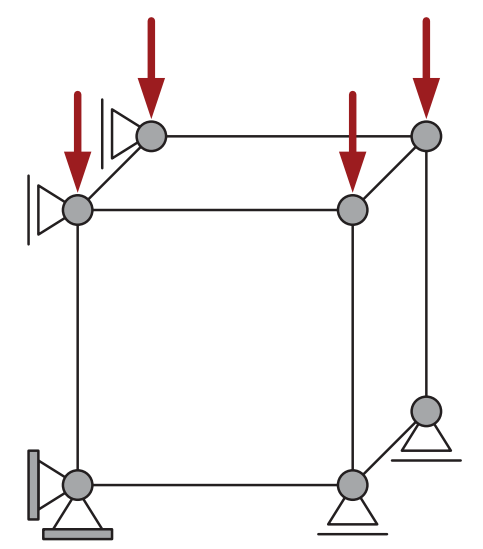

Figure 2. One element setup.

about $110^{-5} 1 / \mathrm{s}$, whereas increasing the strain rates comes with an increasing maximum stress. The resulting uniaxial stress-strain relations in tension and compression are given for sample strain rates in Fig. 3 and Fig. 4, distinguished by material model.

A critical point concerning the VERD model are the high computed strength increases in particular observed in case of compressive loading. This can however be considered of minor importance in the mainly tensile testing configuration of the Split Hopkinson Bar. Also noticeable is the constant stress level the RHT element maintains after having reached its compressive stress peak. The formulations of the KCC and the VERD model on the other hand describe pre-peak nonlinearities as well as post-peak softening. For the given element size and with use of the automatically generated parameters no noteworthy strain rate dependency could be noted for the KCC material model. Computed strength increases with bigger element sizes in case of compressive loading were attributed to inertia effects.

\section{Split Hopkinson Bar modelling}

\subsection{Experimental setup}

Dynamic material response evaluation is continued with the set up of the Split Hopkinson Bar (SHB) [5]. This configuration has widely been used to study the material behavior of concrete under high strain rate conditions.
The classical version of the setup consists of three parts in line. These are the aluminium incident bar, the concrete specimen and the aluminium output bar, which here is omitted for the considered spallation configuration. The loading occurs while a steel impactor hits the incident bar and subjects it to a pulse load. This results in a compressive wave traveling in longitudinal direction through the configuration. With the intension to study the materials tensile response prescribed velocities are used to generate pulse intensities below the concretes compressive strength but above its corresponding tensile value. Considered are pulse intensities after the partial reflection at the contact area between aluminium and concrete. This approach leads to concrete failure shortly after the wave fully reflects at the specimens right free end. Figure 5 schematically shows the course of events.

The SHB analysis is mostly evaluated under the assumption of uniaxial wave propagation, whereas in reality triaxial stress states will takes place. This is encouraged by relatively large specimen diameters, which are necessary in order to counteract the material heterogeneity. This in turn leads to considerable wave form modifications, which is covered by the triaxiality of the material models.

\subsection{Simulation model}

The Split Hopkinson Bar dimensions used for the study correspond to the setup used by Schuler, Mayrhofer \& Thoma (2006) [2]. The diameter of all three parts was thus set to $75 \mathrm{~mm}$. The length was $60 \mathrm{~mm}$ for the steel projectile, $5500 \mathrm{~mm}$ for the incident bar and $250 \mathrm{~mm}$ for the specimen.

Experimental results are given for impactor velocities of $4.1,7.6$ and $11.1 \mathrm{~m} / \mathrm{s}$ respectively. For comparison purposes these initial conditions were also applied in the computations with LS-DYNA.

The material behavior of the steel impactor and the aluminum impactor bar was assumed linear elastic, whereas for the concrete specimen VERD, KCC and RHT material models were taken into account. For the discretization of all three components 8-node single integration point elements $(E L F O R M=1)$ were used. Preliminary numerical results revealed no significant changes below an element size of approximately $6 \mathrm{~mm}$. Modelling was therefore performed with 1050 elements for the projectile, 94500 elements for the impact bar and 4305 elements for the specimen.

\subsection{Results for stresses}

The numerical spallation results are presented by material model. First considered is the KCC model. In the quasistatic case the use of automatically generated material parameters resulted in a quite ductile uniaxial material behavior, as shown in Fig. 3. Regarding the largest compressive stress the first load stage $(4.1 \mathrm{~m} / \mathrm{s})$ resulted in $\approx 28 \mathrm{kN} / \mathrm{m}^{2}$ whereas the largest tensile strength was $\approx 3.4 \mathrm{kN} / \mathrm{m}^{2}$, as shown in Fig. 6 .

The spallation process could potentially be problematically influenced by the ductile material behavior. Cracking is being artificially delayed, whilst further transversal 


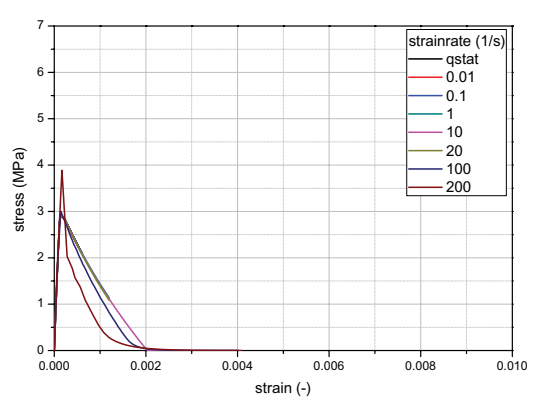

(a) KCC

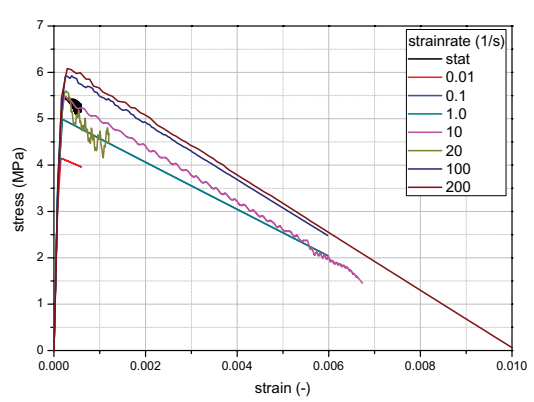

(b) $\mathrm{RHT}$

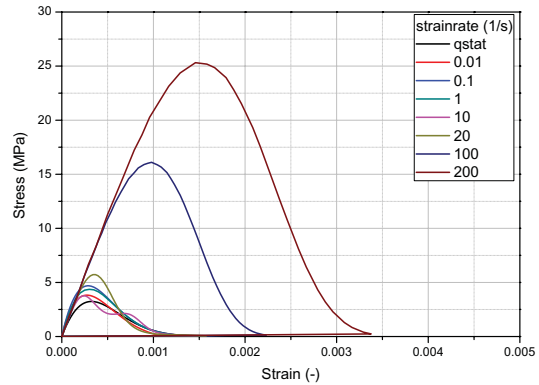

(c) VERD

Figure 3. Uniaxial stress-strain relations for sample strain rates in tension.

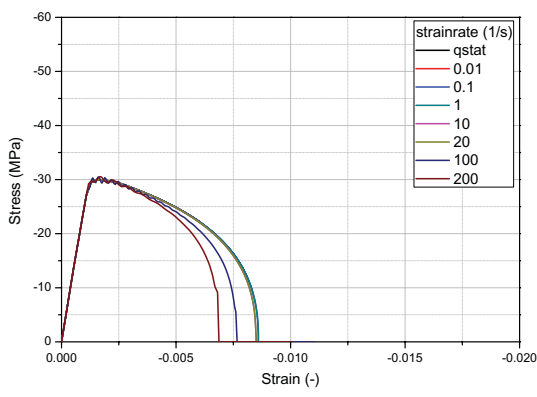

(a) KCC

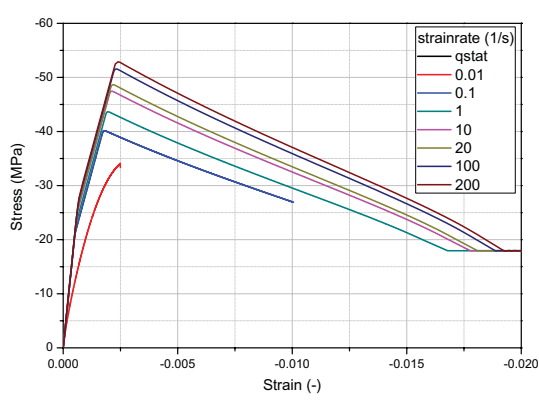

(b) $\mathrm{RHT}$

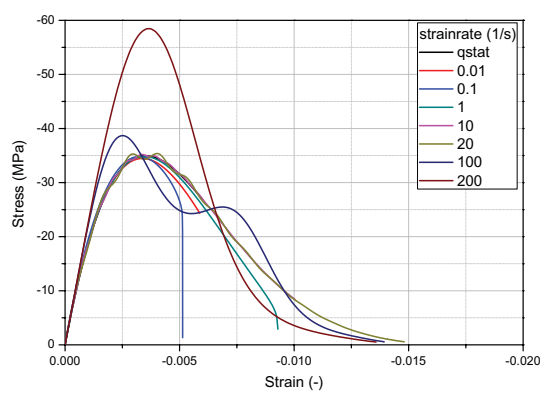

(c) VERD

Figure 4. Uniaxial stress-strain relations for sample strain rates in compression.

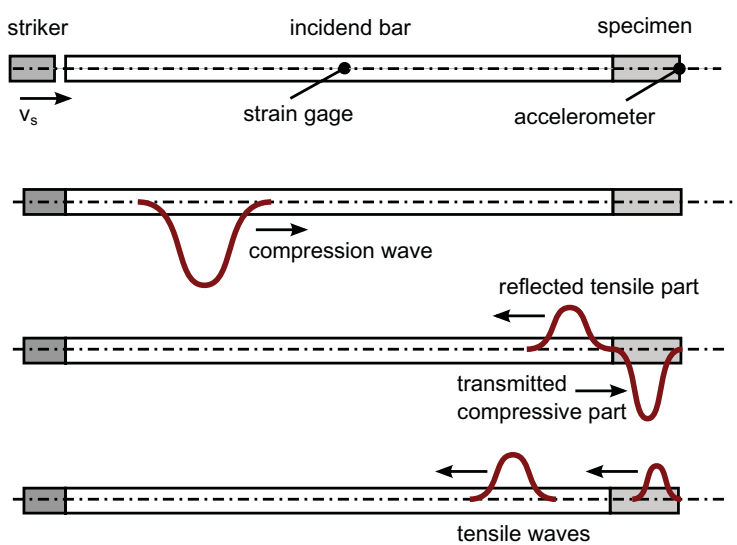

Figure 5. Course of events in the spallation test.

element strain arises from the assumed constant Poissons ratio. This in turn favors multi axial stress states, which affect the elements failure strength.

Loading stage two $(7.6 \mathrm{~m} / \mathrm{s})$ showed similar results in tension and maximum compressive stresses of $\approx 51 \mathrm{kN} / \mathrm{m}^{2}$. The simulation performed with impactor velocity three $(11.1 \mathrm{~m} / \mathrm{s})$ lead to compressive failure of the material. In this cases large permanent deformations are caused by the entry of the compressive pulse, which thus reaches the materials compressive strength. At this point the numerical results do not correspond to the experimental results, where no compressive failure was observed.

Next considered is the RHT model. The study of its response revealed an even more ductile behavior than the KCC material model. The largest compressive stresses increased with increasing projectile velocity and were computed as $\approx 30,53$ and $72 \mathrm{kN} / \mathrm{m}^{2}$. Validation of these

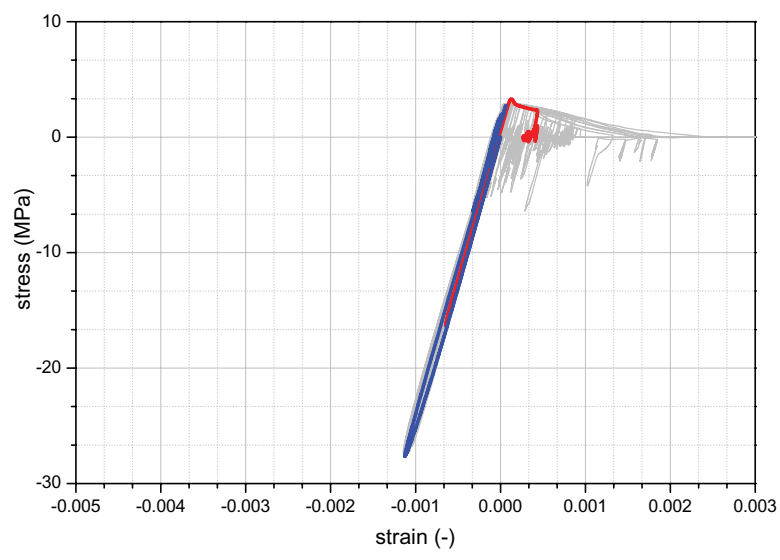

Figure 6. KCC material model: Stress-strain diagram of the specimens centre elements in longitudinal direction for loading stage one.

computations is possible to a limited extent only, since assumed stress states in experimental investigations rely on the adoption of assumed stress strain relations. According to such assumptions maximum compressive stresses were given by Schuler [10] as $\approx 25,49$ and $64 \mathrm{kN} / \mathrm{m}^{2}$ for loading stages one, two and three.

At a closer consideration of loading stage three it becomes apparent that the longitudinal stress strain relations differ strongly from one element to another. This is due primary to the compaction of the porous assumed RHT material and the deviations from the purely longitudinal wave propagation scenario.

Last considered is the VERD material model. Here (for all three loading stages) the maximum tensile strength significantly exceeds the earlier computed stresses, 


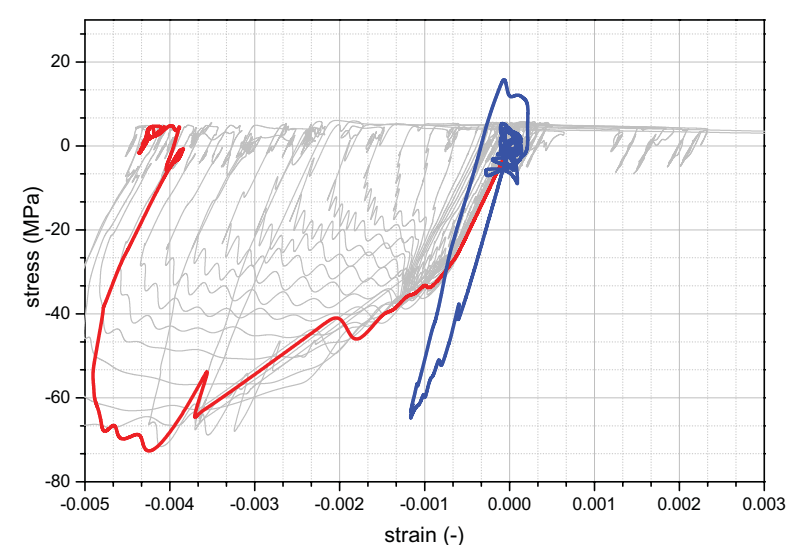

Figure 7. RHT material model: Stress-strain diagram of the specimens centre elements in longitudinal direction for loading stage three.

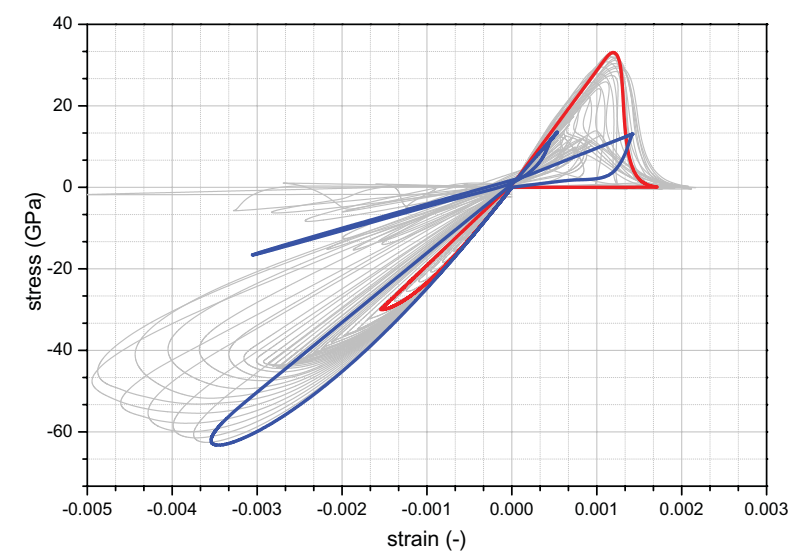

Figure 8. VERD material model: Stress-strain diagram of the specimens centre elements in longitudinal direction for loading stage three.

whereas the maximum compressive stresses come close to the values given by Schuler. Computed were $\approx 25.8,45.6$ and $63.3 \mathrm{kN} / \mathrm{m}^{2}$. Load stage one computes a maximum tensile failure strength of $\approx 25.8 \mathrm{kN} / \mathrm{m}^{2}$, which implies an dynamic increase factor of $\approx 7$ based on the quasistatic value.

\subsection{Results for velocities}

A major point of the Split Hopkinson experiment evaluation considers the determination of the dynamic tensile strength. Since this is experimentally not directly possible an indirect method has been developed based on the velocity data of the right free specimen end.

Particle displacements and velocities arise from the wave entering the specimen as a compression pulse. Provided that its pulse intensity is kept bellow the materials compressive strength tensile failure occurs after the reflection at the specimens free end. It takes place at some distance from the free end and leads to spallation i.e. cracking and fragmentation.

The critical evaluation value is the particle velocity reduction or pull pack velocity $\Delta v_{\mathrm{pb}}$, measured after the first velocity maximum. This value is an indicator for the maximum reached tensile strength inside the specimen.
Basis of the estimation is the uniaxial wave propagation in linear elastic material with brittle tensile fracture. Subject to this requirements the relation

$$
f_{c t, \text { dynamic }}=\frac{1}{2} \rho c \Delta v_{p b}
$$

accurately reflects the failure tensile strength, where $\rho$ is the density of concrete and $c$ the wave speed. In contrast to this physical nonlinearities and multiaxial stress states restrict the validity of the relation, turning it into an estimation.

Focal point of the material model comparison is the evaluation based on the right free end velocity. At this point available experimental data and relatively high measurement accuracy provide a suitable foundation for data valuation. Figure 9 shows the velocities computed for all three material models together with the experimentally recorded ones. It should be noted that velocities of the KCC model are not relevant after compressive failure and are therefore plotted for loading stage one and two only. Following observations can be made:

- Plotting of the right end velocities for the RHT material model results in non-smooth data, which in this case illustrates the deviations from the uniaxial dispersionless wave propagation.

- The VERD model computes the highest free end velocities and the largest pull-back-velocities compared to the other material models. Both measurands are in the same order of magnitude as the corresponding experimental values.

- In this way evaluation of Eq. (1) suggests the highest dynamic tensile stresses for the VERD model. They were calculated as $\approx 11.9,15.4$ and $14.5 \mathrm{kN} / \mathrm{m}^{2}$ in the numerics, whereas Schuler [10] determined the corresponding experimental values to $\approx 12.9,16.2$ and $18 \mathrm{kN} / \mathrm{m}^{2}$.

- Considerations must be given to the fact that while dynamic tensile strengths of the VERD model determinded over equation 1 show good agreement with experimental values, detailed insight into the specimens stress states reveals higher longitudinal stresses (see e.g. Fig. 6).

\subsection{Local insights on the VERD formulation}

Figure 10 is taking a closer look at the specimens behaviour according to the VERD material formulation. The red curve indicates the stress time distribution for the first element carrying the maximum compressive load with underlying greyed average results. The back flip of the stress into the tensile branch shows the relaxing of the free incident surface while the outer pressure load releases. The later oscillating indicates the intactness of the specimens left side. In contrast to this, the blue curve describes the stress distribution at the position of the later failure. Undergoing a compressive load, it reflects on the free right end and induces the tensile cracking at a high stress level.

Figure 11 further extracts this position and illustrates the stress strain relation with its highlighted strain rates distribution. Starting at (0) the point caries compression (1) 


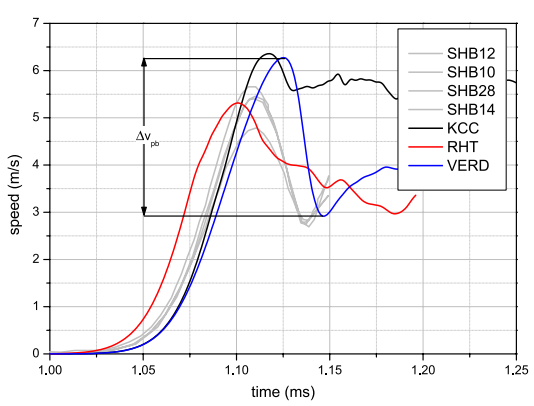

(a) loading stage one

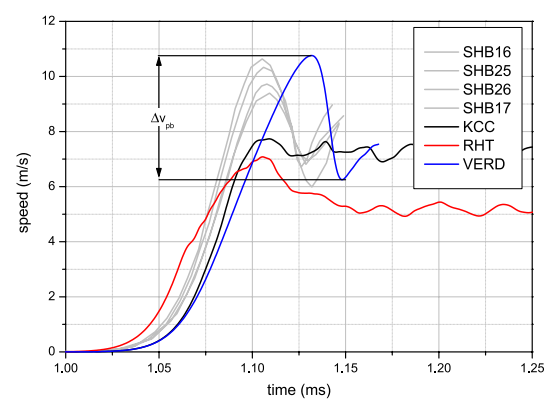

(b) loading stage two

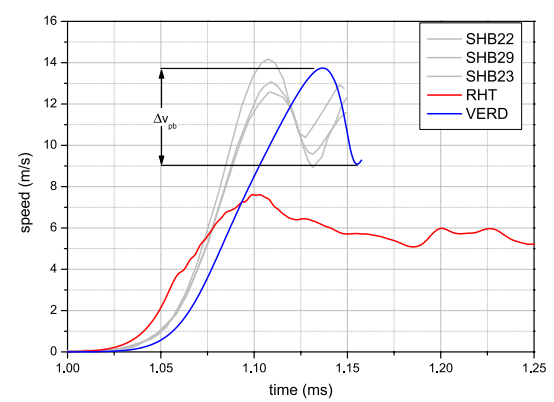

(c) loading stage three

Figure 9. Right free end velocities from computations and experiments.

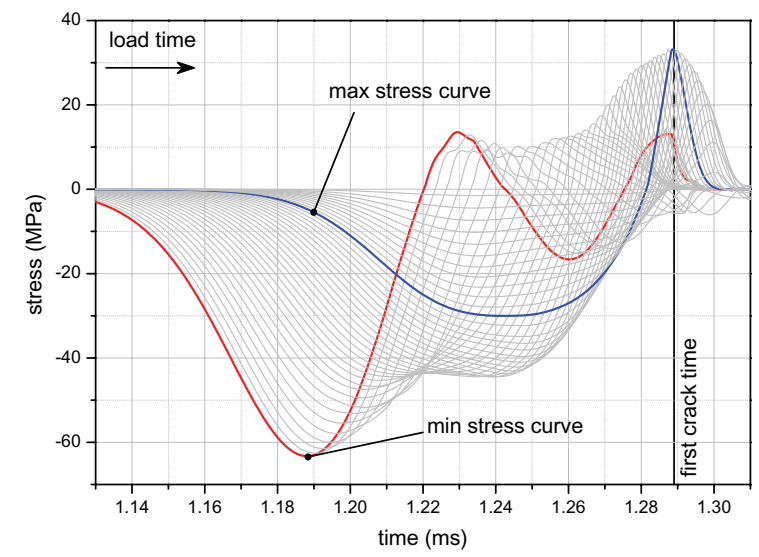

Figure 10. Specimens time based stress distribution.

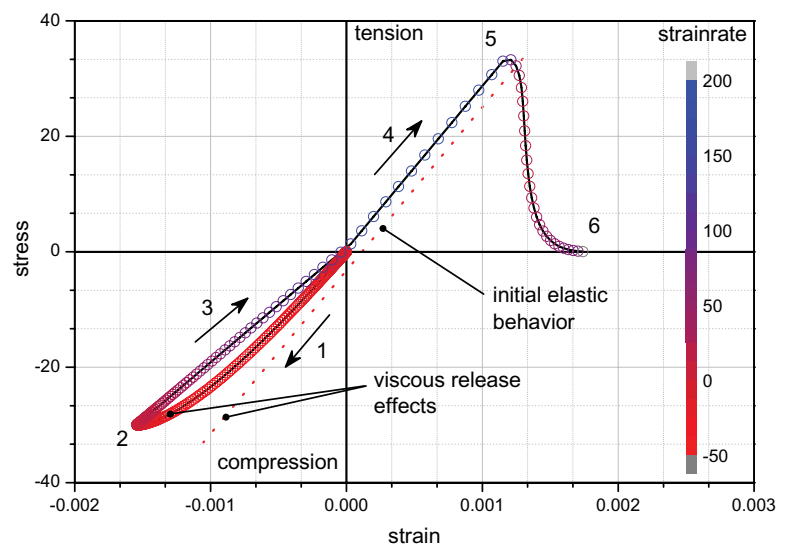

Figure 11. Specimens stress strain relation.

at a moderate strain rate of $\approx 50 \mathrm{~s}^{-1}$. This low rate leads to relaxing elasticity as a result of the viscous formulation and is indicated by the opening gab towards the initial elastic slope. At point (2) the maximum pressure releases and a fast unloading occurs at (3) back to (0). The slope (3) is different to (1) and describes elastic hysteresis rather than damage. This can be shown at the following tensile load. The slope increase in (4) due to the high strain rate of $\approx 200 \mathrm{~s}^{-1}$ and leads to a stiffening with respect to (3). In (5) the tensile stress leads to the damage and stresses relaxes toward zero at (6).

\section{Conclusions}

Performance in simulationing high speed dynamics of concrete was carried out with three different constitutive laws. Examined were the LS-Dyna supported models KCC and RHT and the newly developed material model VERD. Validation was in all three cases carried out by means of relevant experimental data derived from SHB tests in the spallation configuration. Considerable importance is attached to the particle velocities of the right free end of the specimen. The resulting characteristic curves indicate the closest match to the critical velocity decline for the VERD material model. Though better results with proper adjustment of the KCC and RHT material parameters are assumed, the VERD material model achieved superior results regarding the derived failure strength.

Further investigations require variating viscosity and crack retardation related parameters in order to improve the order of magnitude to which computed absolute velocity and strength values match to experimental data.

\section{References}

[1] Häussler-Combe, U.; Kühn, T.: Modelling of Strain Rate Effects for Concrete with Viscoelasticity and Retarded Damage. International Journal of Impact Engineering 50 (2012), 17-28

[2] Schuler, H.; Mayrhofer, C.; Thoma, K.: Spall experiments for the measurement of the tensile strength and fractureenergy of concrete at high strain rates, International Journal of Impact Engineering 32, (2006), 1635-1650

[3] Hussler-Combe, U.: A novel strain-rate model for concrete and its influence on wave. In: Bicanic, N.; Meschke, G.; Mang, H., de Borst, R. (Hrsg.): Proceedings of EURO-C 2010, 24-27 March 2014, St. Anton am Arlberg, Austria., 2014, 97-108

[4] Kolsky, H. (1949). An investigation of the mechanical properties of materials at very high rates of loading. Proc. Physical Society, Section B 62, 676-700

[5] Bischoff, P. \& S. Perry (1991). Compressive behavior of concrete at high strain rates. Materials and Structures 24, 425-450

[6] Malvar, L.J. \& C.A. Ross (1998). Review of strain rate effects for concrete in tension. ACI Materials Journal 95, 735-739 
[7] Rossi, P. (1991) A physical phenomenon which can explain the mechanical behavior of concrete under high strain rates. Materials and Structures 24, 422-424

[8] Reinhardt, H.W. \& J. Weerheijm (1991). Tensile fracture of concrete at high loading rates taking account of inertia and crack velocity effects. International Journal of Fracture 51, 31-42
[9] Eibl, J. \& Schmidt-Hurtienne (1999). Strain-ratesensitive constitutive law for concrete. Journal of Engineering Mechanics 125, 1411-1420

[10] Schuler, H. (2004). Experimentelle und numerische Untersuchungen zur Schdigung von stoßbeanspruchtem Beton (Doctoral dissertation, Universität der Bundeswehr München, Universitätsbibliothek) 